\title{
Assessment of a European Bladder Cancer Predictive Model for Non-Muscle Invasive Bladder Cancer in an Australian Cohort
}

\author{
Matthew Alberto ${ }^{\mathrm{a}, *}$, Stephanie Demkiw ${ }^{\mathrm{a}}$, Jeremy Goad ${ }^{\mathrm{a}}$, Mark Jenkins ${ }^{\mathrm{b}}$, Genevieve Duggan ${ }^{\mathrm{a}}$, \\ Tyler Mow ${ }^{\mathrm{a}}$ and Lih-Ming Wong ${ }^{\mathrm{a}, *}$ \\ ${ }^{a}$ Department of Urology and Surgery, St. Vincent's Hospital Melbourne, Melbourne, VIC, Australia \\ ${ }^{\mathrm{b}}$ The University of Melbourne, Centre for Epidemiology and Biostatistics, Melbourne \\ School of Population and Global Health Parkville, Melbourne, VIC, Australia
}

Received 10 September 2018

Accepted 12 January 2019

\begin{abstract}
.
Background and Objectives: To validate the European Organization for Research and Treatment of Cancer (EORTC) model using an Australian cohort and to identify variables within our cohort that may predict non-muscle invasive bladder cancer (NMIBC) recurrence and progression.

Methods: A retrospective chart review of patients undergoing transurethral resection of bladder tumour (TURBT) at a single academic institution between 1995 and 2015 was performed $(n=366)$. Only patients with available TURBT pathology having initial Ta or T1 disease were included $(n=255)$.

EORTC risk groups were calculated for individual patients and compared to actual recurrence rates using a binomial method comparing observed and expected proportions.

Results: In our cohort of 255 patients, there were 209 men and 46 women, with a median age of 69 years (range 18-93). Intravesical therapy was given to $59 \%(n=152)$. In total, 142 patients $(56 \%)$ experienced cancer recurrence, with median recurrence and progression free survival at 11 months and 25.5 months respectively.

Comparison of EORTC estimates to actual recurrence proportions at 1,3 and 5 years showed the EORTC calculator underestimated the actual recurrence that occurred. However, only EORTC group "score 1-4" estimate was statistically significant compared to the actual recurrence (at 1 year, predicted $24 \%$ vs actual $33 \%, p<0.001$ ).

Conclusion: In validating the EORTC risk calculator in our Australian cohort, we found the calculator underestimated NMIBC recurrence for most of our patients. Longer follow-up time and a larger sample size may assist with validation but true differences in population and treatment may exist. Our results suggest for now, care should be exercised when applying these risk tables to an Australian population.
\end{abstract}

Keywords: Non-muscle invasive bladder cancer, EORTC risk tables, recurrence, Australia

\section{INTRODUCTION}

${ }^{*}$ Correspondence to: Mr Lih-Ming Wong and Dr Matthew Alberto, Department of Urology, St. Vincent's Hospital Melbourne, 41 Victoria Parade, Fitzroy VIC 3065, Australia. E-mails: Lih-Ming.WONG@svha.org.au. and MatthewIrvingAlberto@gmail.com.
Bladder cancer $(\mathrm{BCa})$ poses many challenging clinical issues for management including prevention of recurrence and progression. The majority of $\mathrm{BCa}$ are non-muscle invasive $(75 \%)$ with muscle invasive $(25 \%)$ more likely to progress to life-threatening 
disease [1]. Up to $80 \%$ of pTa non-muscle invasive bladder cancer (NMIBC) patients have recurrence requiring frequent hospital admissions and therefore poses a significant disease burden [2]. NMIBC management usually includes transurethral resection of bladder tumour (TURBT). As an adjunct to resection, intravesical therapy (IVT), with Bacillus Calmette-Guérin (BCG) and Mitomycin (MMC), may be used to decrease risk of recurrence and progression of cancer in the bladder. NMIBC can recur or progress to muscle invasive disease, with 1 year rates ranging from $15-61 \%$ and $<1-17 \%$ respectively [3]. Patients with frequent recurrences have repeated cystoscopy examinations which may significantly impact quality of life. The ability to accurately estimate the risk of recurrence and progression in patients with NMIBC would aid in counselling patients and stratifying intensity of follow-up.

In 2006, the European Organization for Research and Treatment of Cancer (EORTC) developed a simple scoring system to predict risk of NMIBC recurrence and progression [3]. The model provides simple categorization of patients with stratification into low, intermediate, high and very high risk groups based on 6 clinico-pathological factors (grade, T category, number of tumours, concomitant Carcinoma in situ [CIS], tumour size and prior recurrence rate). Probabilities of recurrence and progression at 1 and 5 years were provided after categorization, allowing practitioners to make clear decisions regarding ongoing surveillance and active treatment. The EORTC predictive model is included in the European Association of Urology (EAU) guidelines on NMIBC [4], as well as being easily accessible on the internet [ http://www.eortc.be/tools/bladdercalculator]. However, as the model was derived from a European population, having a higher incidence of bladder cancer, it may not be applicable in other populations such as Australia [5].

Therefore, our primary objective is to validate the model using an Australian cohort, with a secondary objective to identify variables within our cohort which may predict NMIBC recurrence and progression.

\section{MATERIALS AND METHODS}

\section{Study subjects}

A retrospective chart review of all patients undergoing TURBT at a single academic institution between January 1995 and May 2015 was performed $(n=366)$. Ethics approval was granted by our institution's Human Research Ethics Committee. Included patients had a histological diagnosis of urothelial carcinoma and pathological stage Ta or $\mathrm{T} 1$ disease. Urothelial carcinoma of the bladder was based on the TNM classification of the International Union Against Cancer (2009) [6]. Patients without an initial diagnostic TURBT at our institution $(n=61)$ and those with a diagnosis other than Ta or T1 bladder cancer $(n=50)$ were excluded [Supporting Fig. 1]. The total number of patients in our final analysis was 255. Time of diagnosis was classified as the date of the initial TURBT showing NMIBC.

Demographics (age, gender, date of death, cause of death), BCa risk factors (smoking status, occupational exposure, previous cancer diagnoses, chronic inflammation of the bladder and cyclophosphamide use), cystoscopy results, TURBT pathology results (prior recurrence rate, number of tumours, tumour diameter, $\mathrm{T}$ category, tumour grade and concomitant CIS), and exposure to IVT data were collected. The World Health Organization (WHO) system 1973 was used for tumour grade classification [7]. Patients with the WHO 2004 grading system were randomly reclassified according to the MacLennan et al. (2007) conversion system to allow EORTC utilization: low grade papillary urothelial carcinoma (LGPUC), a third were classified into Grade 1, and two thirds into Grade 2 [8-10]. High grade papillary urothelial carcinoma (HPUC) was classified as Grade 3.

Surveillance of patients was via cystoscopy and frequency was dependent on severity of disease based upon the discretion of the treating physician (e.g. Low-risk Ta tumours underwent cystoscopy at 3 months and if negative, repeat examination at 12 months and subsequent annual cystoscopies for 5 years. High-risk tumours underwent cystoscopy and urine cytology at 3 months and if negative, investigations were repeated every 3 months for 2 years then every 6 months until 5 years, and then annually thereafter). Repeat TURBT was performed as required with IVT given on a case by case basis. Mortality and cause of death were obtained from the Victorian Cancer Council.

Patients were defined as having "recurrence" if at any time after diagnosis of initial Ta or T1 bladder disease; the cancer reappeared and had histopathological findings of NMIBC after TURBT (Ta or T1). Patients were defined as having "progression" if at any time after diagnosis of initial Ta or $\mathrm{T} 1$ bladder disease; there were pathological findings of $\mathrm{T} 2$ disease or above after TURBT. That is, progression was 
defined as the change from NMIBC to MIBC. Time to recurrence was defined as from the initial TURBT date to the first documented recurrence. Time to progression was defined as from the initial TURBT date to the first documented progression. Patients were censored at the last available follow-up cystoscopy if they were alive or had died before tumour recurrence.

\section{Statistical analysis}

Our cohort was dichotomized into "recurrence" and "without recurrence" categories for statistical analysis. Due to the limited number of patients undergoing progression $(n=9)$, further analysis was not completed on this subgroup. To assess the accuracy of the EORTC model for recurrence, predicted recurrences were calculated for individual patients based on the EORTC model and compared to actual recurrence rates found in our cohort using the binomial method of comparing observed and expected proportions.

For all patients, the required EORTC 6 clinicopathological variables (prior recurrence rate, number of tumours, tumour diameter, T category, tumour grade and concomitant CIS), were used to generate a total score [Supporting Table 1]. Based on the total score, patients were stratified into four risk groups according to the EORTC recurrence model: low risk (score 0), intermediate risk (score 1-4), high risk (score 5-9), and very high risk (score 10-17). Univariate and multivariate analysis using Cox proportional hazards regression was used to identify baseline predictors of bladder cancer recurrence. Statistical significance was indicated when a $p$ value $<0.05$ was attained and all $p$ values were twosided. All statistical analysis was conducted using STATA/SE 14.0.

\section{RESULTS}

\section{Baseline characteristics}

Characteristics of patients at the time of diagnosis are provided in Table 1 . The median age was 69 years old (range 18-93) with $82.0 \%$ of the cohort male. IVT was given to 152 patients $(59.6 \%)$ with 32 patients (12.6\%) receiving BCG and 120 patients $(47.1 \%)$ receiving MMC (119 [46.7\%] post-operative and 1 [0.3\%] adjuvant course). At diagnosis, most patients had a single tumour $(n=237,92.9 \%)$, were $<3 \mathrm{~cm}$ $(n=233,91.4 \%)$, stage Ta $(n=219,85.9 \%)$ and without concomitant CIS $(n=247,96.9 \%)$. Patients were reclassified from the $2004 \mathrm{WHO}$ grading to the 1973
Table 1

Patient and tumour characteristics

\begin{tabular}{|c|c|}
\hline & $\mathrm{n}(\%)$ \\
\hline Age median (range) & $69(18,93)$ \\
\hline \multicolumn{2}{|l|}{ Gender $(\mathrm{n}, \%) n=255$} \\
\hline Male & $209(81.96 \%)$ \\
\hline Female & $46(18.04 \%)$ \\
\hline \multicolumn{2}{|c|}{ Smoking status at diagnosis (n, \%) $n=259$} \\
\hline Current smoker & $45(17.65 \%)$ \\
\hline Ex-smoker & $94(36.86 \%)$ \\
\hline Never smoked & $34(13.33 \%)$ \\
\hline Unknown & $83(32.55 \%)$ \\
\hline \multicolumn{2}{|l|}{ Prior recurrence rate } \\
\hline Yes, $\leq 1 \mathrm{rec} / \mathrm{yr}$ & $30(11.76 \%)$ \\
\hline Yes, $>1 \mathrm{rec} / \mathrm{yr}$ & $6(2.35 \%)$ \\
\hline No (primary) & $219(85.88 \%)$ \\
\hline Unknown & $0(0.00 \%)$ \\
\hline \multicolumn{2}{|c|}{ Intravesical treatment $n=152$} \\
\hline None & $103(40.39 \%)$ \\
\hline $\mathrm{BCG}$ & $32(12.55 \%)$ \\
\hline Mitomycin & $120(47.06 \%)$ \\
\hline \multicolumn{2}{|l|}{ Number of tumours } \\
\hline 1 & $237(92.94 \%)$ \\
\hline $2-7$ & $18(7.06 \%)$ \\
\hline$\geq 8$ & $0(0 \%)$ \\
\hline \multicolumn{2}{|l|}{ Tumour size } \\
\hline$<3 \mathrm{~cm}$ & $233(91.37 \%)$ \\
\hline$\geq 3 \mathrm{~cm}$ & $18(7.06 \%)$ \\
\hline Unknown & $4(1.57 \%)$ \\
\hline \multicolumn{2}{|l|}{$\mathrm{T}$ category } \\
\hline $\mathrm{Ta}$ & $219(85.88 \%)$ \\
\hline $\mathrm{T} 1$ & $36(14.12 \%)$ \\
\hline \multicolumn{2}{|l|}{ 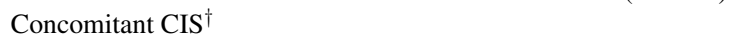 } \\
\hline No & $247(96.87 \%)$ \\
\hline Yes & $8(3.14 \%)$ \\
\hline \multicolumn{2}{|l|}{ Grade } \\
\hline G1 & $45(17.65 \%)$ \\
\hline $\mathrm{G} 2$ & $153(60.00 \%)$ \\
\hline G3 & $56(21.96 \%)$ \\
\hline Unknown & $1(0.39 \%)$ \\
\hline \multicolumn{2}{|l|}{ G3T1 } \\
\hline No & $247(96.86 \%)$ \\
\hline Yes, with CIS & $2(0.78 \%)$ \\
\hline Yes, no CIS & $26(10.20 \%)$ \\
\hline
\end{tabular}

WHO grading as the EORTC calculator utilised the latter system. All LGPUC $(n=140)$ were randomly assigned into one third Grade $1(n=45)$ and two thirds Grade $2(n=95)$ based on recent comparisons of the two grading systems [8-10].

The median length of follow up for the cohort was 44 months (IQR 12-92). Overall, 142 patients (56.0\%) experienced recurrence and 9 patients $(3.5 \%)$ had progression. The median recurrence free survival was 10 months (IQR 4-31) with a median progression free survival of 25.5 months (IQR 7-52). Our cohort had 64 deaths (25.1\%) with 14 cases $(5.5 \%)$ due to bladder cancer. 


\section{Validation of the EORTC risk calculator}

Comparison of the EORTC predicted recurrence to the actual recurrence in our cohort at 1 year is shown in Table 2 . For the 1 year calculation, most of our patients $(n=222,91.73 \%)$ were in EORTC predicted groups "score 0" and "score 1-4". There were only 19 patients $(7.85 \%)$ in "score $5-9$ " and 1 patient $(0.41 \%)$ in "score $10-17$ ". Statistically significant differences were found when the EORTC group "score 1-4" estimate was compared to the actual recurrence in our cohort at 1 year (predicted 24\% vs actual $33 \%$ (95\% CI 28-43), $p<0.001$ ). Analysis was also completed at 3 and 5 years [Supporting Table 2 and 3] and statistically significant differences for group "score 1-4" was similarly seen with 3 year follow up (3 years predicted $40 \%$ vs actual $62 \%$ (95\% CI 45-77), $p=0.008)$. Therefore, the EORTC calculator underestimated the actual recurrence for patients in the lowest scoring risk groups (score 0 and score $1-4)$.

Table 3 contains univariate and multivariate analysis of our cohort. Univariate analysis for predictors of NMIBC recurrence showed age (HR 1.02 (1.00-1.04), $p=0.03$ ) and $\mathrm{T}$ category (HR 1.91 $(1.21-3.02), p=0.006)$ to be significant. In multivariate analysis, only tumour grade was significant (G2 vs G1 HR $1.61(1.07-2.42), p=0.022)$.

\section{DISCUSSION}

We believe that this study is the first to examine validity of the EORTC predictive model for NMIBC recurrence in an Australian cohort. Our results [Table 2] showed the EORTC predictive model significantly underestimated the risk of recurrence of NMIBC in our cohort of predominantly "low risk" bladder cancer at 1,3 and 5 year follow up. Our results, in comparison to other external validation studies of the EORTC model are presented in Table 4. Whilst small in number compared to the original Sylvester et al. [3] paper $(n=2596)$, our cohort is similar in size to other previous publications $(n=91$ to 417$)$.

The 1-year recurrence rate found in our cohort for low risk NMIBC was underestimated by the model. It is higher than the original EORTC cohort (15\%), and other publications (0-13.7\%) [Table 4]. Comparison of our results to the EORTC cohort of pathological features at baseline TURBT suggests fundamental differences between our cohorts: incidence of primary tumours (86\% vs EORTC 54\%), number of tumours (single tumours; 93\% vs EORTC 56\%), T category (Ta; $86 \%$ vs EORTC $56 \%$ ), tumour grade (G1; $18 \%$ vs EORTC $43 \%$, G2; 60\% vs EORTC $44 \%$ and $\mathrm{G} 3 ; 22 \%$ vs EORTC $10 \%$ ) and tumour size $(<3 \mathrm{~cm} ; 91 \%$ vs EORTC $80 \%)$. The IVT rates in our Australian cohort were 59\% compared to $78 \%$ in the EORTC cohort, but other publications varied in proportion of patients receiving IVT (19.3-100\%), and the type of IVT used (Doxorubicin, Pirarubicin and BCG). Whilst our cohort has more low risk patients at baseline TURBT, we report a higher than expected recurrence rate at 1 year. Our study was designed to examine reasons for recurrence, including: incomplete resection, tumour cell re-implantation, growth of microscopic tumours, and new tumour formation [11]. While the first two are influenced by the clinician pre- and immediately post treatment, the latter two are influenced by chemoprevention. Metaanalysis for MMC show decreased rate of recurrence [12], however novel studies have suggested continuous irrigation with sterile water is a cheaper option with no statistical difference in recurrence-free rate [13].

It should be noted that the EORTC study analysed patients treated between 1979-1989; and hence, treatment was not in accordance with the contemporary guidelines. Other contemporary validation studies have varying pathological features and differing treatment regimens, so comparison of results is difficult. For cohorts where all patients received intravesical BCG $[14,15]$, recurrence rates might be lower and the EORTC calculator is likely to underestimate. However, the well-known issues of compliance, due to complications and adverse events also need consideration [16]. Inherent problems with EORTC classification system are well recognized. Stratification dependent on a size threshold e.g. size of tumour $(2.9 \mathrm{~cm}-0$ points versus $3.0 \mathrm{~cm}-3$ points $)$, is open to inter-observer errors, bias and documentation errors [17]. The size threshold simplifies usage but likely sacrifices accuracy for variables which we know are continuous. Several other studies note similar issues with implementation of the EORTC tables [14, 18-21].

Our cohort demonstrated differences from the EORTC cohort. In particular, despite multiple risk factors suggesting lower risk (grade, stage, single tumour, tumour size $<3 \mathrm{~cm}$ ) our recurrence rate was higher ( $56 \%$ vs $48 \%)$. This might be explained by our smaller retrospective sample size or errors in reclassification, but it is possible that other differences outside of the EORTC risk factors may exist. Other 
Table 2

Exact (binomial method) comparing observed proportion vs proportion expected based on EORTC

\begin{tabular}{|c|c|c|c|c|c|c|}
\hline \multirow{2}{*}{$\begin{array}{l}\text { EORTC 1 year } \\
\text { Recurrence } \\
\text { groups }\end{array}$} & \multicolumn{2}{|c|}{ Recurrence within 1 year } & \multirow[t]{2}{*}{ Total } & \multirow{2}{*}{$\begin{array}{c}\text { EORTC } 1 \text { year } \\
\text { predicted } \\
\text { percentage } \\
\text { recurrence }[95 \% \mathrm{CI}]\end{array}$} & \multirow{2}{*}{$\begin{array}{c}\text { Actual } \\
\text { percentage } \\
\text { recurrence }[95 \% \mathrm{CI}]\end{array}$} & \multirow[t]{2}{*}{$p$-value $(*)$} \\
\hline & No & Yes & & & & \\
\hline Score 0 & 25 & 7 & 32 & 15 [10-19] & $22[9-40]$ & 0.32 \\
\hline Score $1-4$ & 123 & 67 & 190 & $24[21-26]$ & $33[28-43]$ & $<0.001$ \\
\hline Score 5-9 & 11 & 8 & 19 & $38[35-41]$ & $40[20-67]$ & 0.81 \\
\hline Score $10-17$ & 0 & 1 & 1 & $61[55-67]$ & 100 & 1.00 \\
\hline Grand Total & 159 & 83 & 242 & & & \\
\hline
\end{tabular}

*Exact binomial method.

Table 3

Univariate and Multivariate analysis of time to recurrence (Cox $\left.\mathrm{PH}^{*}\right)$

\begin{tabular}{|c|c|c|c|c|}
\hline \multirow[t]{2}{*}{ Variable } & \multicolumn{4}{|c|}{$\mathrm{HR}^{* *}$ of non-recurrence $(n=114)$ versus recurrence $(n=141)$} \\
\hline & Univariable $[95 \% \mathrm{CI}]$ & $p$-value & Multivariable $[95 \% \mathrm{CI}]$ & $p$-value \\
\hline Age & $1.02[1.00,1.04]$ & 0.030 & $1.02[1.00,1.05]$ & 0.089 \\
\hline Gender: male, female & $1.11[0.72,1.72]$ & 0.639 & $1.01[0.60,1.69]$ & 0.972 \\
\hline \multicolumn{5}{|l|}{ Number of tumours } \\
\hline 1 (reference) & - & - & - & - \\
\hline $2-7$ & $1.07[0.88,1.33]$ & 0.467 & $1.11[0.83,1.49]$ & 0.460 \\
\hline$\geq 8$ & $\mathrm{n} / \mathrm{a}$ & $\mathrm{n} / \mathrm{a}$ & $\mathrm{n} / \mathrm{a}$ & $\mathrm{n} / \mathrm{a}$ \\
\hline Tumour size: $<3 \mathrm{~cm}, \geq 3 \mathrm{~cm}$ & $1.15[0.93,1.41]$ & 0.197 & $1.13[0.86,1.48]$ & 0.376 \\
\hline T category: Ta, T1 & $1.91[1.21,3.02]$ & 0.006 & $1.24[0.46,3.31]$ & 0.668 \\
\hline CIS $^{\dagger}:$ no, yes & $1.40[0.57,3.47]$ & 0.454 & $1.30[0.30,5.53]$ & 0.724 \\
\hline \multicolumn{5}{|l|}{ Grade } \\
\hline 1 (reference) & - & - & - & - \\
\hline 2 & $1.04[0.64,1.70]$ & 0.868 & $1.61[1.07,2.42]$ & 0.022 \\
\hline 3 & $1.48[0.85,2.58]$ & 0.171 & $0.82[0.33,2.00]$ & 0.666 \\
\hline
\end{tabular}

${ }^{*}$ Cox Proportional Hazards Regression. ${ }^{* *}$ Hazards ratio. ${ }^{\dagger}$ Carcinoma in situ.

well-known risk factors not included in the EORTC risk calculator noted by the EUA which may account for population differences include tobacco smoking and occupational exposure (aromatic amines, polycyclic aromatic hydrocarbons and chlorinated hydrocarbons) [4]. Due to the retrospective nature of our study, this data was not available to us. Furthermore, EAU guidelines also note that genetic predisposition may have an influence on bladder cancer incidence indirectly through susceptibility to other risk factors [4]. There may be specific genes that are yet to be identified which may explain the lower Australian rates for risk factors based on the EORTC calculator contrasted with higher recurrence rates. Finally, differences in treatment could account for differences in recurrence. In our cohort only $59 \%$ of our patients received intravesical therapy compared to $78 \%$ in the EORTC group (Table 4). Therefore, underestimation by the EORTC model may be due to influences outside of the clinico-pathologic risk factors.

In our cohort, Grade was identified as an independent predictor for time to recurrence. This is consistent with reviewed literature [3, 20, 21-24], however other variables (gender, number of tumours, tumour size and CIS) were not found to be significant and are likely to be explained by our relatively small sample size. Other important variables include postresection chemotherapy, maintenance therapies and timing of repeat endoscopic resection. Buethe and Sexton (2011) reviewed the literature and noted that these additional characteristics may have influence on the endpoints of multivariate analysis, however despite an apparent association may not provide additional accuracy to the EORTC model when added [25].

It remains debatable whether the newer WHO 2004 definitions demonstrate greater prognostic value [4]. As our grade of cancer classification crossed both WHO 1973 and WHO 2004 systems we required conversion. Our literature review found that many previous validation studies did not account for changes in pathology grading [14, 15, 18, 20-23]. As formal histopathological review was not performed, we accounted for changes in grading system by utilising the methods espoused by Grignon (2009) and MacLennan et al. (2007). All LGPUC $(n=140)$ were randomly assigned into one third Grade $1(n=45)$ 


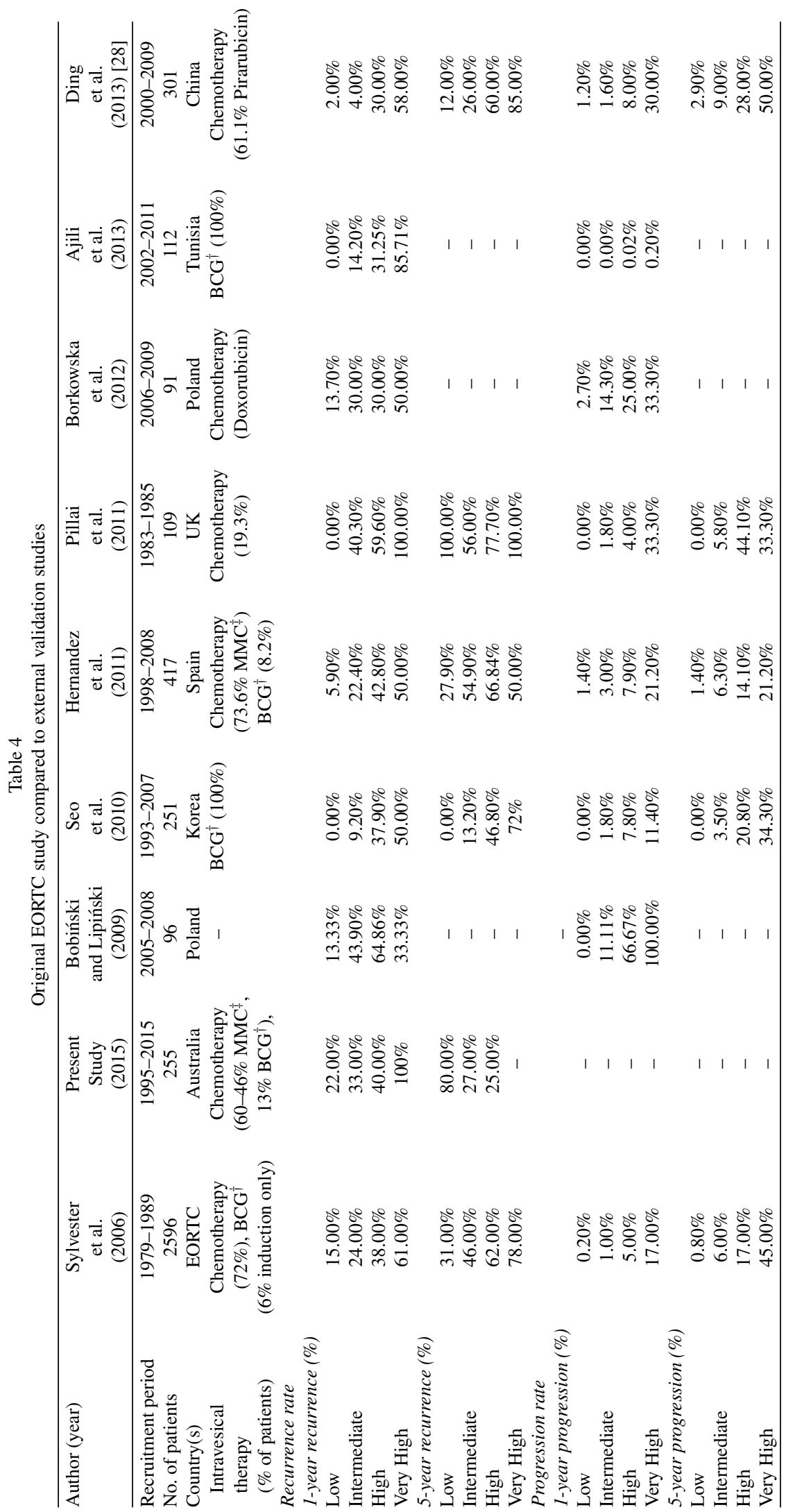


and two thirds Grade $2(n=95)$ based on recent comparisons of the two grading systems [8-10]. However, MacLennan et al. (2007) questioned their interchangeability as the original WHO 1973 morphologic descriptions were "vague and subjective" compared to the newer WHO 2004 definitions which emphasized the three new categories have clear cut definitions. Additionally, the frequency of Grade 1 WHO 1973 disease in our cohort is likely underestimated given data for papillary urothelial neoplasm of low malignant potential (PUNLMP) was not collected. Furthermore, the WHO 2004 classification considers all T1 tumours as high grade. The number of T1 patients was 36, with 28 being Grade 3 $(n=28 / 36,78 \%)$ and this could represent further evidence of variation in tumour grading.

Our cohort had a single patient stratified into the "very high" EORTC category (0.4\%). Other studies report a range of $2-14 \%$ of their cohorts with "very high" risk: 7\% EORTC, 10\% (Seo et al. 2010), 14\% (CUETO 2011), 4\% (Sakano et al. 2010), 2\% (Borkowska et al. 2013). Hence, our cohort may be a different (less aggressive) one compared to the EORTC population, or this may simply be reflecting our small study population, or errors from the reclassification process. We also note that our patients were not managed with the latest EAU guidelines, including re-resection to detect residual tumour and understaging. And subsequently there would be a significant effect on recurrence-free survival [27]. Further data on re-resection would improve accuracy of recurrence rates.

To conclude, we found that the EORTC calculator underestimated the NMIBC recurrence risk for patients with "score 1-4". Differences in our cohort to that from which the EORTC calculator was derived may reflect our small sample size, but true differences may exist and further validation in larger Australian cohorts would be useful. For now, care should be exercised when applying the EORTC risk tables to an Australian population.

\section{CONFLICT OF INTEREST}

The authors have no conflict of interest to report.

\section{SUPPLEMENTARY MATERIAL}

The supplementary material is available in the electronic version of this article: http://dx.doi.org/ 10.3233/BLC-180199.

\section{REFERENCES}

[1] Battista Di Pierro G, Gulia C, Cristini C, Fraietta G, Marini L, Grande P, et al. Bladder Cancer: A Simple Model Becomes Complex. Current Genomics. 2012;13(5):395415.

[2] van Rhijn B, Burger M, Lotan Y, Solsona E, Stief C, Sylvester R, et al. Recurrence and Progression of Disease in Non-Muscle-Invasive Bladder Cancer: From Epidemiology to Treatment Strategy. European Urology. 2009;56(3):43042.

[3] Sylvester R, van der Meijden A, Oosterlinck W, Witjes J, Bouffioux C, Denis L, et al. Predicting Recurrence and Progression in Individual Patients with Stage Ta T1 Bladder Cancer Using EORTC Risk Tables: A Combined Analysis of 2596 Patients from Seven EORTC Trials. European Urology. 2006;49(3):466-77.

[4] Babjuk M, Bohle A, Burger M, Comperat E, Kaasinen E, Palou J, et al. EAU guidelines on Non-muscle-invasive Bladder Cancer (Ta, T1 and CIS): Update 2015. European Urology. 2015;4-27.

[5] Chavan S, Bray F, Lortet-Tieulent J, Goodman M, Jemal A. International Variations in Bladder Cancer Incidence and Mortality. European Urology. 2014;66(1):59-73.

[6] Sobin L, Gospodariwicz M, Wittekind C. TNM Classification of Malignant Tumours. 2009;262-5.

[7] Epstein J, Amin M, Reuter V, Mostofi F. The World Health Organization/International Society of Urological Pathology Consensus Classification of Urothelial (Transitional Cell) Neoplasms of the Urinary Bladder. The American Journal of Surgical Pathology. 1998;22(12):1435-48.

[8] MacLennan GT, Kirkali Z, Cheng L. Histologic grading of noninvasive papillary urothelial neoplasms. European Urology. 2007;51(4):889-97.

[9] Samaratunga H, Makarov DV, Epstein JI. Comparison of WHO/ISUP and WHO classification of noninvasive papillary urothelial neoplasms for risk of progression. Urology. 2002;60:315-19.

[10] Yin H, Leong AS-Y. Histologic grading of noninvasive papillary urothelial tumors: Validation of the 1998 WHO/ISUP system by immunophenotyping and follow up. Am J Clin Pathol. 2004;121:679-87.

[11] Bryan R, Collins S, Daykin M, Zeegers M, Cheng K, Wallace $\mathrm{D}$, et al. Mechanisms of recurrence of $\mathrm{Ta} / \mathrm{T} 1$ bladder cancer. The Annals of The Royal College of Surgeons of England. 2010;92(6):519-24.

[12] Chou R, Selp S, Buckley DI, et al. Intravesical Therapy for the Treatment of Nonmuscle Invasive Bladder Cancer: A Systematic Review and Meta-Analysis. The Journal of Urology. 2017;197(5):1189-99.

[13] Bijalwan P, Kumar Pooleri G, Thomas A, Comparison of sterile water irrigation versus intravesical mitomycin $\mathrm{C}$ in preventing recurrence of nonmuscle invasive bladder cancer after transurethral resection. Indian Journal of Urology. 2017;33(2):144-8.

[14] Seo K, Kim B, Park C, Kim C, Chang H. The Efficacy of the EORTC Scoring System and Risk Tables for the Prediction of Recurrence and Progression of Non-Muscle-Invasive Bladder Cancer after Intravesical Bacillus Calmette-Guerin Instillation. Korean Journal of Urology. 2010;51(3):165.

[15] Ajili F, Darouiche A, Chebil M, Boubaker S. The Efficiency of the EORTC Scoring System for the Prediction of Recurrence and Progression of Non-muscle-invasive Bladder Cancer Treated by Bacillus Calmette-Guerin Immunotherapy. Ultrastructural Pathology. 2013;37(4):249-53. 
[16] Lamm DL, Blumenstein BA, Crissman JD, et al. Maintenance bacillus Calmette-Guerin immunotherapy for recurrent TA, T1 and carcinoma in situ transitional cell carcinoma of the bladder: A randomized Southwest Oncology Group Study. J Urol. 2000;163(4):1124-9.

[17] Mian CPycha A. Risk Stratification in Superficial Bladder Cancer: Fact or Fiction. EAU-EBU Update Series. 2007;5(2):63-9.

[18] Fernandez-Gomez J, Madero R, Solsona E, Unda M, Martinez-Piñeiro L, Ojea A, et al. The EORTC tables overestimate the risk of recurrence and progression in patients with non-muscle-invasive bladder cancer treated with bacillus Calmette-Guérin: External validation of the EORTC risk tables. International Braz j Urol. 2011;37(5):671-2.

[19] van Rhijn B, Zuiverloon T, Vis A, Radvanyi F, van Leenders G, Ooms B, et al. Molecular Grade (FGFR3/MIB1) and EORTC Risk Scores Are Predictive in Primary Non-Muscle-Invasive Bladder Cancer. European Urology. 2010;58(3):433-41.

[20] Sakano S, Matsuyama H, Takai K, Yoshihiro S, Kamiryo $\mathrm{Y}$, Shirataki S, et al. Risk group stratification to predict recurrence after transurethral resection in Japanese patients with stage Ta and T1 bladder tumours: Validation study on the European Association of Urology guidelines. BJU International. 2010;107(10):1598-604.

[21] Borkowska E, Jȩdrzejczyk A, Marks P, Catto J, Kałużewski B. EORTC risk tables - their usefulness in the assessment of recurrence and progression risk in non-muscle-invasive bladder cancer in Polish patients. Central European Journal of Urology. 2013;65:14-20.
[22] Pillai R, Wang D, Mayer E, Abel P. Do Standardised Prognostic Algorithms Reflect Local Practice? Application of EORTC Risk Tables for Non-Muscle Invasive (pTa/pT1) Bladder Cancer Recurrence and Progression in a Local Cohort. The Scientific World JOURNAL. 2011;11:751-9.

[23] Hernández V, De La Peña E, Martin M, Blázquez C, Diaz F, Llorente C. External validation and applicability of the EORTC risk tables for non-muscle-invasive bladder cancer. World Journal of Urology. 2010;29(4):409-14.

[24] Millan-Rodriguez F, Chechile-Toniolo G, Salvador-Bayarri J, Palou J, Algaba F, Vicente-Rodriguez J. Primary Superficial Bladder Cancer Risk Groups According to Progression, Mortality and Recurrence. The Journal of Urology. 2000;680-4

[25] Buethe D, Sexton W. Bladder cancer: Validating the EORTC risk tables in BCG-treated patients. Nature Reviews Urology. 2011;8(9):480-1.

[26] Grignon D. The current classification of urothelial neoplasms. Modern Pathology. 2009;22:S60-S69.

[27] Babjuk M, Bohle A, Burger M, Comperat E, Kaasinen E, Palou J, et al. EAU guidelines on Non-muscle-invasive Bladder Cancer (TaT1 and CIS): Update 2017. European Urology. 2017;4-27.

[28] Ding W, Chen Z, Gou Y, Sun C, Xu K, Tan J, et al. Are EORTC risk tables suitable for Chinese patients with nonmuscle-invasive bladder cancer? IJCEDP. 2014. 